\section{Poverty and environmental stress fuel Darfur crisis}

SIR - The "true culprit" in the Darfur crisis is the National Islamic Front, according to two recent News stories ('Darfur's climate roots challenged' Nature 447, 1038; 2007, and 'Darfur lake is a "mirage" Nature 448, 394-395; 2007).

Political scientists understandably point to specific parties, leaders and military factions to account for a specific crisis such as Darfur. It is also understandable that they do not want to make excuses for perpetrators of violence. Still, as quoted, they have gone too far in the opposite direction. They seem to overlook the point that Darfur's extreme poverty, rising population, growing water stress and desertification are all important contributors to the Darfur crisis.

The most authoritative recent study of the subject - the United Nations Environment Programme's report Sudan: Post-Conflict Environmental Assessment (www.unep.org/ sudan) - rightly notes that desertification, land degradation and climate change are major factors in the crisis, and appropriately comments that they are "generally contributing factors only, not the sole cause for tension". The report also states that long-term peace in the region will become possible only if environmental and livelihood issues are resolved.

These findings are in line with a growing number of studies that find extreme poverty, falling incomes and failing rains are strong predictors of outbreaks of violence in Africa. An excellent new book Too Poor for Peace? (eds L. Brainard and D. Chollet, Brookings Institution Press, 2007) notes that recent research strongly favours the claim that these are the crucial drivers of conflict in less developed countries; much less solid evidence implicates political repression.

It is important that considerations of ecology, climate and extreme poverty are integrated with political analyses of conflict. This is especially the case as demographic pressures hit against resource limitations and climate change in the Horn of Africa and elsewhere.

Jeffrey D. Sachs

The Earth Institute at Columbia University, 535 West 116th St, New York, New York 10027, USA

\section{A paper should appear with all the information it needs}

SIR - Until the past few years, both Nature and Science confined their articles and letters to a rather small number of words. This was both good and bad; good in that the articles were short and to the point; bad in that it eliminated studies that were complex. I first thought that the Supplementary Information sections were a great idea. Here was a way to place at the readers' disposal important data (tables or figures) that were necessary background to the work, but not necessary to the reading and understanding of the paper.

However, some recent articles refute my thinking. One or two have contained tens of pages of this supplementary material, essential to the reading and understanding of the article. Ten pages of Supplementary Information are not unusual, and the average for Nature is about five pages.

I suggest either that you either publish hard-copy papers whole and integrated in a long form, or publish them whole and integrated on the web, as you now do with Methods sections.

Larry Benson

Chief Arid Regions Climate Project, USGS Suite E127, 3215 Marine St,

Boulder, Colorado 80303-1066, USA

Comments are welcomed at Nautilus: http://blogs.nature.com/nautilus/2007/09/ papers_should_not_need_supp.html

\section{Diverse approaches useful for microbicide trials}

SIR - Your News story 'HIV trial doomed by design, say critics' (Nature 448, 110-111; 2007), portrays a biased and excessively pessimistic view of the Centre for the AIDS Programme of Research in South Africa (CAPRISA) 004 trial. As the first microbicide trial to be initiated from, and partially funded by, South Africa, the CAPRISA 004 trial promises to address some fundamental questions about the safety and effectiveness of tenofovir as a microbicide gel.

More published data exist on tenofovir for HIV prevention than for any other microbicide; thirteen monkey and four human tenofovir studies were considered when designing the CAPRISA 004 trial.

In the rural South African community where CAPRISA runs a large AIDS treatment programme, women with migrant partners account for most new HIV infections. The CAPRISA 004 trial and its episodic dosing strategy of two doses in 24 hours, one before and one after intercourse, was designed to maximize product adherence and hence the presence of tenofovir at exposure, especially for women with migrant partners.

This dosing strategy was selected after extensive consultation with international scientific experts, including those knowledgeable about microbicides, tenofovir and clinical-trial design. Just as importantly, it followed detailed consultation with the communities involved. Moreover, to assess the effectiveness of this strategy, extensive data will be collected on gel use, timing of gel use in relation to intercourse, adherence to the dosing regimen and tenofovir levels.

Differences of opinion and interpretation of data are important in science, including research on women-initiated HIV prevention, as you note in your Editorial 'Transmission lines' (Nature 448, 225-226; 2007). But in the absence of a marker that can show whether a particular agent, dosing strategy or trial design will lead to protection against HIV, only the trial results will reveal the truth. Salim S. Abdool Karim, Quarraisha Abdool Karim CAPRISA, Doris Duke Medical Research Institute, Nelson R. Mandela School of Medicine, University of KwaZulu-Natal, Durban, South Africa

\section{DAMA sheds light on dark-matter particles}

SIR - We were disconcerted to see that the experiment XENON10 was the main focus of your News Feature 'Welcome to the dark side' on searches for particle dark matter (Nature 448, 240-245; 2007), even though it is still in its infancy. The well-established DAMA/NaI experiment, on the other hand, was confined to a box titled 'Contested results'.

Experiments searching for dark-matter particles must be able to discriminate between a dark-matter signal and background signals created by other particles. Because the number of dark-matter particles hitting Earth is expected to vary during the course of a year, one strategy is to look for an annual variation (with many specific features) in the frequency of peculiar events registered by a detector. Running successfully for seven years, the DAMA/NaI experiment, with its 100-kilogram sodium iodide target, has detected such a peculiar annual variation, the properties of which meet those expected of a dark-matter signal with a confidence level of more than six standard deviations.

It has been proved quantitatively that no effect could produce this signal apart from dark-matter particles. Also, the DAMA effect is not contradicted by the results of other experiments because these experiments take a different approach, and so are unable to investigate the same effect.

The DAMA effect has been proved to be compatible with the most popular darkmatter candidate: the neutralino. Moreover, the DAMA experiment is sensitive to physical scenarios and candidates other than the neutralino - to which other experiments are either poorly sensitive or even blind.

Rita Bernabei ${ }^{\star}$ (on behalf of the DAMA Collaboration), Alessandro Bottino' *Department of Physics, University of Rome Tor Vergata, and INFN Rome Tor Vergata, via della Ricerca Scientifica 1, I-00133 Rome, Italy

Department of Theoretical Physics, University of Torino, and INFN Torino, via P. Giuria 1, I-10125 Torino, Italy 